Karen E. A. Burns

Celia Zubrinich

John Marshall

Deborah Cook

\section{The 'Consent to Research' paradigm in critical care: challenges and potential solutions}

Received: 15 April 2009

Accepted: 5 June 2009

Published online: 7 July 2009

(C) Springer-Verlag 2009

K. E. A. Burns ( $)$ C C. Zubrinich · J. Marshall

Interdepartmental Division of Critical Care Medicine,

St Michael's Hospital, St. Michael's Hospital,

University of Toronto, Toronto, ON M5B-1W8, Canada

e-mail: burnsk@smh.toronto.on.ca

Tel.: +1-416-8646060

Fax: +1-416-8646013

K. E. A. Burns - J. Marshall

Keenan Research Centre and the Li Ka Shing Knowledge Institute, St. Michael's Hospital, Toronto, ON, Canada

D. Cook

Department of Clinical Epidemiology and Biostatistics, McMaster University, Hamilton, ON, Canada

Clinical research in the intensive care unit (ICU) poses unique challenges. Investigators must operationalize protocols under emergency conditions, recruit patients within narrow time windows, study illnesses with high morbidity and mortality, and obtain consent for enrolment of critically ill patients who, by virtue of their illness, usually lack autonomous decision-making capacity. Difficulties in obtaining informed consent may reduce opportunities for critically ill patients to participate in research, prolong study implementation, limit the generalizability of study results, and delay identification of potentially effective, ineffective, and harmful interventions.

The requirement for informed consent to participate in research arises from the ethical principle of autonomy. Respect of this principle requires that individuals understand the study and provide consent to receive a research intervention and for use of their personal health information [1]. However, critically ill patients are rarely able to comprehend or communicate clearly. Consequently, consent for research involving critically ill patients typically requires substituted judgments provided by a substitute decision maker (SDM) or legal representative.

The identification of SDMs for and the conduct of research using alternate consent models in patients lacking decision-making capacity varies across jurisdictions. Legal representatives are designated according to predefined hierarchies in Canada [2], Italy, Spain and The Netherlands, through provisions made under European laws in several European countries and by the judicial system in Germany [3]. In the United Kingdom, clinicians obtain assent for research participation from either a personal legal representative or, in their absence, a professional legal representative (e.g., an independent physician or a person nominated by the most responsible healthcare provider) [3]. While research can be conducted under a waiver of consent in Scotland (http://www.opsi. gov.uk/si/si2006/20062984.htm) and in many European countries (Belgium, Germany, France, The Netherlands), waived consent is not permitted in others (Poland, Portugal, Italy, Denmark), and requires community consultation in the United States. Regulations passed in Australia in 2007 enable research to be conducted in unconscious patients only if it is "designed both to be therapeutic for them and to improve treatment for the condition from which they suffer." (http://www.nhmrsc. gov.au/publications/synopses/_files/e72.pdf). Notwithstanding, decision makers other than SDMs seldom provide consent for research participation and Research Ethics Boards (REBs) infrequently waive consent to participate in critical care studies.

The families of critically ill patients are overwhelmed by the ICU environment, the gravity and prognostic uncertainty regarding their loved one's condition, and the imminent threat of death of their family member. 
Nowhere in medicine do health care providers, patients and families interact with such intensity and ambiguity, often for protracted periods of time. For patients unable to give informed consent, debate exists over whether consent should always be required and, if so, to whom the decision regarding participation in research should be entrusted. Alternative models to SDM consent include deferred consent (deferred to patient, deferred to SDM), two physician consent, request for an 'objection to consent', and waived consent (or exemption from informed consent). Although contemporary consent models for participation in acute care research are grounded in efforts to respect patient autonomy, protect potentially vulnerable populations and ensure the ethical conduct of research, their application may have important consequences for SDMs, the care provided to critically ill patients, and the results of studies in the ICU setting.

A combination of the complexity of the information and the stress inherent in making decisions for a gravely ill loved one renders the integrity of the decision-making process uncertain. A survey of SDMs providing consent for therapeutic measures revealed that more than half of SDMs failed to understand information presented to them regarding diagnosis, treatment and prognosis [4]. No comparable investigations have been conducted to evaluate SDMs' comprehension regarding research participation.

Moreover, the perspectives of the SDM and the patient may be discordant. A systematic review, including 151 scenarios involving 2,595 surrogate-patient pairs, assessed SDMs' ability to predict patients' treatment preferences. SDMs predicted patients' treatment preferences with $68 \%$ accuracy. The accuracy of their substituted judgments did not vary according to health state or intervention, by prior discussion of the involved parties or SDM type (patient-designated vs. legally designated) [5]. In a study by Coppolino and colleagues, patients agreed or declined to provide consent to two hypothetical trials (minimal-risk and greater-than-minimal-risk) and surrogates attempted to predict patients' responses. The authors found an overall surrogate positive predictive value of $85 \%$ for the low-risk study and $80 \%$ for the higher-risk study. The authors also found that SDM consent resulted in a 'false positive consent rate' of 16-20\%, with SDMs frequently making decisions that differ from those patients would have made [6]. Similarly, in a prospective multicentre study evaluating decisions to participate in research at ICU discharge, Ciroldi and coworkers compared patients, SDMs, and physicians' wishes to participate in a minimal-risk and a greater-thanminimal-risk scenario. Whereas SDMs underestimated patients' wishes regarding participation in both the minimal and greater-than-minimal-risk scenarios, physicians overestimated the patients' desire to participate in lowrisk scenarios and underestimated patients' wishes to participate in the greater-than-minimal-risk scenario. Regardless of the scenario, SDMs expressed the highest uncertainty regarding patient participation in research [7]. SDMs are clearly an imperfect surrogate for the autonomous decision of the patient.

Substituted judgments may not only fail to accurately represent patients' wishes, but may cause duress for SDMs that may be greater in magnitude than that experienced in making treatment decisions. In one study, being asked to provide consent to research was associated with post-traumatic stress symptoms in $35 \%$ of family members of critically ill patients with $9 / 18(50 \%)$ and 33/69 (48\%) of individuals involved in discussions about appropriate level of care and consent to research experiencing these symptoms, respectively [8]. In contrast, discussions and decisions about clinical care, such as performing a tracheostomy and regarding appropriate level of care, induced substantially lower rates of posttraumatic symptoms, at 6 and 10\%, respectively.

The challenges of the informed consent process threaten the duration of studies and the generalizability of their results. An internet based survey of Canadian Critical Care Trials Group (CCCTG) members involving 26 ICUs (containing 609 beds and 2,698 admissions) during April 2005, revealed that only 136 adult or pediatric patients, representing $5 \%$ of all admissions, were recruited into an observational or interventional study [9]. In a recent survey to examine implementation challenges, Rose et al. contacted CCCTG investigators who completed eight large randomized controlled trials (RCTs) enrolling 6,658 patients. While the predicted mean trial duration was 3.0 years, the actual trial enrolment period was significantly longer and the anticipated mean monthly recruitment rate tended to be higher than the actual monthly recruitment [10]. While the precise contribution of lack of informed consent is not certain for the foregoing issues, these survey findings highlight the difficulties encountered in implementing critical care research under the SDM paradigm. As a result of these challenges, some investigators have opined that "a more liberal approach is needed to allow for trial participation in patients who cannot offer first person consent" [11].

Since federal rules in the United States were passed in 1996 requiring public disclosure to implement studies without consent, there has been an estimated 3-5-fold decrease in the number of studies involving emergency patients in the United States [12]. While the Food and Drug administration received 56 applications requesting exemption from informed consent between 1996 and 2006, only 21 studies were implemented [12]. In a recent editorial, Mitka and colleagues state that "many of the standard protocols for treating patients with emergency conditions have not been tested in rigorous trials ... instead [they] rely on clinician experience, judgment and tradition" and "fear of approving trials (with alternate consent models) for patients unable to give consent ... is a disservice to this population" [12]. While the federal regulations were developed to protect patients, some 
researchers and ethicists advocate for a broader definition of 'protecting' acutely ill patients which also grants them access to experimental procedures and therapies that may favorably impact their outcome [12].

Alternate consent models appear to be congruent with patient preferences. In a trial of 300 septic patients randomized to hydrocortisone and fludrocortisone or placebo for 7 days, Annane and colleagues increased the enrolment rate from four patients per month (before a waiver of consent was approved) to ten patients per month after approval. In this trial, consent was obtained from next of kin $(23 \%)$ and patients (3\%) a minority of the time, and in $74 \%$ using waived consent [13]. Of those enrolled under waived consent and approached for first party consent (assent), only one patient did not wish to participate and was withdrawn from the study and its analyses. The change in admissible approaches to consent during trial implementation was not only acceptable to study subjects, but also improved the low enrolment rate and enabled timely completion of this landmark trial. In an RCT of a pulmonary artery catheter use in critically ill patients in 56 centers, only $3 \%$ of patients provided first party consent. Following randomization, the research team sought relative assent by asking available relatives if the patient “would have objected to taking part?" Relative assent was obtained in 394/485 (82\%). Of the 181 survivors (including 139 patients for which relative agreement was obtained and 42 for which no relative agreement was obtained) approached for retrospective consent after regaining competency, only $6(3 \%)$ (1 with relative agreement; 5 without relative agreement) declined and were subsequently withdrawn [14]. In interviews involving 240 survivors of critical illness, Scales and coworkers identified that while most participants found the usual practice of SDMs providing consent for enrollment to be acceptable, approximately 15-25\% considered forgoing informed consent to be acceptable and only a minority considered delayed consent to be unacceptable across all study scenarios [15].

To address the challenges associated with the current consent paradigm, potential solutions may involve tailoring SDM consent models to the risks (low, moderate, and high-risk) posed by individual study protocols, including more frequent use of waived consent or alternative consent paradigms (deferred consent, two physician consent, request for an 'objection to consent') in low-risk studies, adoption of hybrid consent models within and among trials, and permitting appropriate types of research to be conducted through other mechanisms when SDMs are unavailable or nonexistent.

\section{Conclusion}

Investigators, REBs, hospitals and granting agencies share responsibility to ensure the conduct of high quality, safe, scientifically rigorous, and ethically sound research. While participant safety and well-being are paramount, critically ill patients should not be denied the opportunity to benefit from clinical research participation and deserve timely identification of beneficial, non-beneficial and harmful interventions. Advocacy of the critical care community regarding the need for alternative consent paradigms and adoption of hybrid consent models in critical care research is required. Additional empirical investigations are also required to better understand the decisional burden experienced by SDMs and to further the debate over the 'consent to research' paradigm in critical care.

Conflict of interest statement No author has a financial relationship with a commercial entity that has an interest in the subject of the manuscript.

\section{References}

1. The Belmont report: ethical principles and guidelines for the protection of human subjects of research (1979) Report of the National Commission for the Protection of Human Subjects of Biomedical and Behavioral Research. Commissioned by the Department of Health, Education and Welfare 9:44 Fed Reg 23192

2. Health Canada (November 25, 2002) Canada Health Act Overview (2002) About health Canada. Canada, Retrieved on December 16, 2008
3. Lemaire F (2007) Emergency research: only possible if consent is waived? Curr Opin Crit Care 13:122-125

4. Azoulay E, Chevret S, Leleu G, Pochard F, Barboteu M, Adrie C, Canoui P, Le Gall JR, Schlemmer B (2000) Half the families of intensive care patients experience inadequate communication with physicians. Intensive Care Med 28:3044-3049

5. Shalowitz DI, Garrett-Mayer E, Wendler D (2006) The accuracy of surrogate decision makers. Arch Intern Med 166:493-497
6. Coppolino M, Ackerson L (2001) Do surrogate decision makers provide accurate consent for intensive care research? Chest 119:603-612

7. Ciroldi M, Cariou A, Adrie C, Annane D, Cohen Y, Delahaye A, Joly LM, Galliot R, Garrouste-Orgeas M, Papazian L, Michel F, Barnes NK, Schlemmer B, Pochard F, Azoulay E, Famirea study group (2007) Ability of family members to predict patient's consent to critical care research. Intensive Care Med 33:807-813 
8. Azoulay E, Pochard F, Kentish-Barnes N, Chevret S, Aboab J, Adrie C, Annane D, Bleichner G, Bollaert PE, Darmon M, Fassier T, Galliot R, Garrouste-Orgeas M, Goulenok C, Goldgran-Toledano D, Hayon J, Jourdain M, Kaidomar M, Laplace C, Larché J, Liotier J, Papazian L, Poisson C, Reignier J, Saidi F, Schlemmer B, FAMIREA Study Group (2005) Risk of post-traumatic stress symptoms in family members of intensive care unit patients. Intensive Care Med 171:987994

9. Marshall J, Cook DJ (2009) Investigator-led clinical research consortia: the Canadian critical care trials group. Crit Care Med 37:S165S172
10. Rose L, Burns KEA, Smith O, Marshall J (2009) Quantifying threats to the feasibility of clinical trails in critically ill patients. Am J Respir Crit Care Med 157:A41-A1580

11. Cook DJ, Moore-Cox A, Xavier D, Lauzier F, Roberts I (2008)

Randomized trials in vulnerable populations. Clin Trials 0:1-9

12. Mitka M (2007) Aiding emergency research aim of report on exemptions to informed consent. JAMA 298:26082609

13. Annane D, Outin H, Fisch C, Bellissant E (2004) The effect of waiving consent on enrollment in a sepsis trial. Intensive Care Med 30:321-324
14. Harvey SE, Elbourne D, Ashcroft J, Jones CM, Rowan K (2006) Informed consent in clinical trials in critical care: experience from the PAC Man study. Intensive Care Med 32:2020-2025

15. Scales DC, Smith OM, Barrett JK, Baum M, Li A, Kraus S, Lutz K, McDonald E, Rosenberg J, Chapman K, Pinto R, Ratnapalan M, Soliven J, Friedrich JO, Lazar NM, Cook DJ, Ferguson ND for the Canadian Critical Care Trials Group (2007) Patient evaluations rating methods for inclusion in trials (PERMIT) pilot study. Am J Respir Crit Care Med 175:B97-A508 